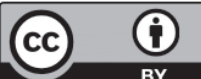

https://creativecommons.org/licenses/by/4.0/

\title{
DESARROLLO DE UN PLAN ESTRATÉGICO EN UNA INSTITUCIÓN DE SALUD QUE OFRECE SERVICIOS ONCOLÓGICOS A TRAVÉS DE UN ESTUDIO DE COMPETENCIAS
}

\section{The development of a strategic plan within a health institution that offers oncology services through an assessment of competencies}

NELSON STEVEN ROMERO ROJAS ${ }^{1}$, JUAN DAVID ESTRADA RODRÍGUEZ², M.B.A. ING. EVER FUENTES ROJAS ${ }^{3}$

Recibido:01 de noviembre de 2018. Aceptado:12 de diciembre de 2018

DOI: http://dx.doi.org/10.21017/rimci.2019.v6.n11.a61

\begin{abstract}
Resumen
En la actualidad, la elaboración de los planes estratégicos no se puede basar únicamente con los datos estadísticos del comportamiento que tienen las diferentes instituciones, sino que deben tener en cuenta cuales son los procesos de evolución que vienen presentando al interior del sector en el que está interactuando. Debido a esto, se desarrolla un plan estratégico para una entidad de salud basado en un estudio de competencias que tiene como objetivo determinar la posición de los servicios en el mercado como eje central para la planeación estratégica de la organización. Se utilizaron herramientas de ingeniería que contribuyeron a la formulación y al mejoramiento de las metas que permitan el liderazgo y posicionamiento en las entidades oncológicas. Finalmente, se generaron escenarios que permitieron priorizar las estrategias y obtener como resultado el plan estratégico sistémico para la gestión de los próximos cuatros años en la Institución.
\end{abstract}

Palabras clave: competencias, escenario, estrategias, mejoramiento, mercado.

\begin{abstract}
At present, the development of strategic plans cannot be based solely on the statistical data of the behaviour of the different institutions, but must take into account the evolution processes that they are presenting within the sector in which they are interacting. Because of this, a strategic plan for a health entity is developed based on a competency study that aims to determine the position of services in the market as a central axis for the strategic planning of the organization. Engineering tools were used that contributed to the formulation and improvement of goals that allow leadership and positioning in oncological entities. Finally, scenarios were generated that allowed to prioritize the strategies and obtain as result the systemic strategic plan for the management of the next four years in the Institution.
\end{abstract}

Keywords: competences, scene, strategies, improvement, market.

\section{INTRODUCCIÓN}

La Planificación Estratégica, es una herramienta de gestión que permite apoyar la toma de deci- siones de las organizaciones en torno al quehacer actual y al camino que deben recorrer en el futuro para adecuarse a los cambios y a las demandas que les impone el entorno y lograr la mayor

1 Ingeniería industrial, Estudiante de la Faculta de Ingeniería, Universidad Libre Bogotá - Colombia.

2 Ingeniería industrial, Estudiante de la Facultad de Ingeniería, Universidad Libre Bogotá - Colombia.

3 MBA., profesor de la Facultad de Ingeniería, Universidad Libre Bogotá - Colombia. ID: https://orcid.org/0000-0001-9671-5884 Correo Electrónico: ever.fuentes@unilibre.edu.co 
eficiencia, eficacia, calidad en los bienes y servicios que se proveen[1].

Todas las organizaciones requieren de una planeación de las actividades, servicios y los recursos que ofrecen para lograr los objetivos y metas trazados. En el caso específico de la entidad que se está trabajando en este proyecto, la planeación se realiza con base en los proyectos de las áreas y los informes de gestión, lo que no permite evidenciar de forma correcta el escenario real del sector de tratamientos oncológicos, por lo tanto, no se puede sustentar a largo plazo[2].

Por medio de herramientas ingenieriles que se aplicaron a los directores de cada una de las áreas, se obtuvo un panorama más claro de los problemas internos que tenía la Institución. Para el análisis externo se creó una matriz basada en las 10 P's del mercadeo, la cual evalúa cada uno de los ámbitos en las diferentes instituciones, para identificar cuál es el posicionamiento de la institución con respecto a las demás, generando así estrategias que fortalezcan los procesos de elaboración de metas y objetivos corporativos, las cuales fueron priorizadas y analizadas a través de la generación de prospectiva por escenarios[3].

Por último, se desarrolló un plan estratégico, que integra todas las áreas (financiera, calidad, control interno)[4][5], que facilita la consecución de los objetivos por parte de todos los empleados, con el fin de que éstos tengan una visión hacia el mismo horizonte, se comprometan y logren el crecimiento y competitividad de la Institución.

\section{Estrategia metodológica}

Se determinó que el estudio de caso fue de tipo aplicado, ya que permitió la aplicación de herramientas de ingeniería para la solución de una problemática concreta y real. El proceso metodológico que se estableció fue: Diagnóstico, a través de técnicas específicas que ayudaron a la recolección de información como la observación, las entrevistas y los cuestionarios. Análisis, se desarrollaron matrices ingenieriles que permitieron evidenciar el estado actual de la Institución y entrar a dar una solución con respecto a los problemas encontrados en la planeación estratégica. Resultados, donde se generaron y evaluaron las estrategias con base en el estudio de competencias que permiten generar el Plan Estratégico de la Institución.

En la etapa de diagnóstico, se realizó la revisión de antecedentes (informes, documentos, artículos y proyectos elaborados por otros investigadores) que permitió tener como referente para el proceso de desarrollo, el estudio elaborado por Claudia Alvarado en una Institución dedicada a la Salud Ocupacional, que aportó la metodología para diseñar el DOFA y con ello generar oportunidades de mejora[6]. El objetivo fue mejorar los resultados de la compañía, tanto monetarios como de gestión, reorganizando los procesos inmersos en el área operativa, administración financiera, comercial, recursos humanos, entre otros[7].

Un factor diferenciador del proyecto fue generar la planeación estratégica basada en un estudio de competencias que permitiera evaluar el sector de salud en el área de oncología en diferentes aspectos, para ellos se tomó como base el estudio realizado por Jhonny Ballesteros, donde aplica las 4 P's del marketing (producto, precio, plaza, promoción)[8][9]. Sin embargo, como aporte para el proceso de investigación, se trabajaron las 10 P's de marketing que permiten tener una visión más amplia de las capacidades internas y externas de las entidades que participaron en el proceso durante el año 2018.

\section{Resultados}

A lo largo del tiempo, la institución se ha posicionado en la mente de los clientes ofreciendo los servicios de oncología, consulta externa y servicio farmacéutico. Esto le ha permitido sostenerse en el tiempo, sin embargo, la evolución del mercado hace que se involucren sus fluctuaciones en los procesos de planeación estratégica, generación de objetivos y sus canales de comunicación, pero no se tiene definida una metodología apropiada para ello. A continuación, se presentan los resultados más importantes del desarrollo de cada objetivo planteado:

\section{A. Análisis interno}

El análisis se fundamentó en la aplicación de 3 herramientas dentro de la Institución, la primera 
es la matriz PESTEL, que sirve para comprender el crecimiento o declive de un mercado, y, en consecuencia, la posición, potencial y dirección de un negocio. Evalúa los aspectos políticos, económicos, sociales, tecnológicos, ambientales y legales que tienen un impacto en el desarrollo de esta[10]. El aspecto en el que la Institución se encontró mejor avaluada fue el económico como se evidencia en la Tabla I.

El aspecto más bajo con respecto a los demás fue el tecnológico, debido a que la Institución no cuenta con un área de TIC que permita, entre otros aspectos la protección de los datos de los clientes y trabajadores.

La segunda fue la matriz de gobierno corporativo, la cual da a conocer el estado en el que se encuentran las relaciones entre la administración, su consejo administrativo y otras partes interesadas, con el fin de determinar los aspectos de la Institución que no están por el rango que se desea[11][12]. Además, poder cuantificar el nivel de cumplimiento de cada criterio a evaluar y hacer el respectivo análisis de cada uno de estos, los resultados obtenidos se pueden ver en la Tabla II.

La tabla anterior indica que el nivel de cumplimiento total de la institución es del $45,91 \%$ sobre un $100 \%$, es decir un nivel medio. El nivel de cumplimiento de cada ámbito es la división del porcentaje de relevancia sobre el nivel total $(100 \%)$, multiplicado por el porcentaje de cumplimiento, en el primer criterio el resultado fue $14,13 \%$.
El tercero fueron las encuestas aplicadas a todos los trabajadores que fueron seleccionados por el área de Calidad con el propósito de identificar cómo perciben ellos el direccionamiento de la Institución y si conocen los aspectos relacionados a la planeación y los objetivos organizacionales. Los resultados obtenidos fueron muy positivos, los empleados se sentían identificados con las políticas, principios y valores de la Institución, además del trato que les dan a ellos.

\section{B. Estudio de competencias}

Uno de los resultados obtenidos del estudio de competencias fue la matriz de perfil competitivo, la cual evidencia que la Institución ocupa el tercer lugar de las siete instituciones analizadas teniendo en cuenta el servicio, demostrando que el funcionamiento ha sido adecuado y se distingue entre la competencia (ver Tabla III).

Luego, teniendo en cuenta los mismos factores de éxito, se generaron doce estrategias que se proponen a la Institución para alcanzar el liderazgo en el sector de la prevención y tratamiento del cáncer.

A continuación, en la Tabla IV se muestran parte de los resultados obtenidos:

Para medir el impacto que tendrían las estrategias en la Institución se desarrolló una matriz de Leopold en la que se cruzó cada una de las estrategias con cada uno de los aspectos de la PESTEL, el

Tabla I. Fragmento de la matriz del aspecto económico.

\begin{tabular}{|c|c|c|c|c|c|}
\hline Económico & $\begin{array}{c}\text { En total } \\
\text { desacuerdo }\end{array}$ & $\begin{array}{l}\text { No está de } \\
\text { acuerdo }\end{array}$ & $\begin{array}{l}\text { Está de } \\
\text { acuerdo }\end{array}$ & $\begin{array}{l}\text { Está bastante } \\
\text { de acuerdo }\end{array}$ & $\begin{array}{l}\text { En total } \\
\text { acuerdo }\end{array}$ \\
\hline $\begin{array}{l}\text { ¿Las expectativas de crecimiento económico generales } \\
\text { afectan crucialmente al mercado donde operamos? }\end{array}$ & & & & & $X$ \\
\hline $\begin{array}{l}\text { ¿La globalización permite a nuestro sector gozar de } \\
\text { importantes oportunidades en nuevos mercados? }\end{array}$ & & & & & $x$ \\
\hline $\begin{array}{l}\text { ¿La tasa de desempleo en Colombia afecta a la } \\
\text { Institución en cuanto a su actividad económica y } \\
\text { desarrollo del sector? }\end{array}$ & & & $x$ & & \\
\hline $\begin{array}{l}\text { ¿Las expectativas del ciclo económico de nuestro sector } \\
\text { impactan en la situación económica de sus empresas? }\end{array}$ & & & & & $X$ \\
\hline $\begin{array}{l}\text { ¿La reforma tributaria está generando repercusiones en } \\
\text { la Institución por ser sin ánimo de lucro? }\end{array}$ & & & & & $X$ \\
\hline
\end{tabular}

Fuente: Los autores, 2018. 
Tabla II. Resultados matriz gobierno corporativo.

\begin{tabular}{|c|c|c|c|}
\hline Criterios de evaluación & $\begin{array}{l}\text { Porcentaje de relevancia } \\
\text { general asignado }(\%)\end{array}$ & $\begin{array}{l}\text { Porcentaje de cumplimiento } \\
\text { alcanzado del ámbito ( } \%)\end{array}$ & $\begin{array}{l}\text { Nivel de cumplimiento } \\
\text { de cada ámbito ( } \%)\end{array}$ \\
\hline $\begin{array}{l}\text { 1. Información de socios } \\
\text { o accionistas, } \\
\text { administradores y } \\
\text { junta directiva }\end{array}$ & 25 & 57 & 14,13 \\
\hline $\begin{array}{l}\text { 2. Transparencia de la } \\
\text { información }\end{array}$ & 20 & 40 & 8,00 \\
\hline $\begin{array}{ll}\text { 3. } & \text { Responsabilidad social } \\
\text { empresarial }\end{array}$ & 25 & 33 & 8,15 \\
\hline $\begin{array}{l}\text { 4. Productos, clientes y } \\
\text { proveedores }\end{array}$ & 15 & 79 & 11,88 \\
\hline $\begin{array}{l}\text { 5. Prácticas antifraude y } \\
\text { corrupción }\end{array}$ & 15 & 25 & 3,75 \\
\hline 6. TOTAL & 100 & & 45,91 \\
\hline
\end{tabular}

Fuente: Los autores, 2018

Tabla III. Matriz de perfil competitivo.

\begin{tabular}{|l|c|c|c|c|c|c|c|}
\cline { 3 - 8 } \multicolumn{2}{c|}{} & \multicolumn{2}{c|}{ Institución } & \multicolumn{2}{c|}{ Competencia 4 } & \multicolumn{2}{c|}{ Competencia 7 } \\
\hline $\begin{array}{l}\text { Factores críticos para } \\
\text { el éxito }\end{array}$ & Peso & Calificación & $\begin{array}{c}\text { Calificación } \\
\text { ponderada }\end{array}$ & Calificación & $\begin{array}{c}\text { Calificación } \\
\text { ponderada }\end{array}$ & Calificación & $\begin{array}{c}\text { Calificación } \\
\text { ponderada }\end{array}$ \\
\hline $\begin{array}{l}\text { Participación en el } \\
\text { mercado }\end{array}$ & 0,05 & 6 & 0,3 & 7 & 0,35 & 8 & 0,4 \\
\hline $\begin{array}{l}\text { Atención a niños y } \\
\text { adultos }\end{array}$ & 0,1 & 4 & 0,4 & 7 & 0,7 & 4 & 0,4 \\
\hline $\begin{array}{l}\text { Servicio farmacéutico } \\
\text { con central de mezclas }\end{array}$ & 0,1 & 5 & 0,5 & 5 & 0,5 & 10 & 1 \\
\hline $\begin{array}{l}\text { Talento humano } \\
\text { especializado }\end{array}$ & 0,05 & 8 & 0,4 & 8 & 0,4 & 8 & 0,4 \\
\hline $\begin{array}{l}\text { Servicio humanizado y } \\
\text { de agrado para el } \\
\text { paciente }\end{array}$ & 0,05 & 6 & 0,3 & 6 & 0,3 & 6 & 0,3 \\
\hline $\begin{array}{l}\text { Seguimiento a los } \\
\text { pacientes }\end{array}$ & 0,05 & 7 & 0,35 & 9 & 0,45 & 7 & 0,35 \\
\hline TOTAL & $\mathbf{1 , 0 0}$ & 79 & $\mathbf{6 , 3}$ & 87 & 6,95 & 96 & 7,4 \\
\hline
\end{tabular}

Fuente: Los autores, 2018

Tabla IV. Estrategias propuestas.

\begin{tabular}{|c|l|}
\hline Número & \multicolumn{1}{|c|}{ Estrategia } \\
\hline $\mathbf{1}$ & Destacarse en el mercado por su atención a niños y adultos, siendo de las pocas que lo realicen \\
\hline $\mathbf{2}$ & Tener cobertura a nivel nacional \\
\hline $\mathbf{3}$ & Crear alianzas con hospitales, para operar como unidad funcional \\
\hline
\end{tabular}

Fuente: Los autores, 2018 
principal objetivo de la matriz fue garantizar que las estrategias tuvieran un impacto positivo en el desarrollo del plan estratégico. En la Tabla V, se muestra una síntesis de los resultados obtenidos.

En esta matriz, se concluye que el impacto de las estrategias generadas es positivo, de 690 puntos el resultado fue 647 , indicando que las estrategias propuestas tendrán el efecto que se espera en el plan estratégico.

\section{Prospectiva}

La prospectiva es la ciencia del futuro, se enfoca a la importancia de la visión estratégica que debe servir a toda organización como parte fundamental de visión de futuro organizacional[11]. Para la evaluación de la prospectiva, se realizó la investigación de la incidencia del cáncer en Colombia, frente a hombres y mujeres y el crecimiento que ha tenido en los últimos años. Se consultó la información de Globocan para el año 2018[12][13].
Una vez terminada la investigación, se identificaron los factores críticos para el desempeño del tratamiento y prevención del cáncer, en la Tabla VI se muestra una parte de estos.

Luego se clasificaron las variables en tecnológicas y no tecnológicas, para evaluarlas según su participación en el mercado, calidad del servicio, el precio de los servicios y costos de operación. Las variables que están por encima de tres, resultan ser influyentes en cada uno de los aspectos analizados, en una escala de cero a cinco. Los resultados se observan en los gráficos 1 y 2 .

Es importante señalar que la totalidad de las variables identificadas fueron consideradas críticas de acuerdo con su impacto en el proceso, es por ello que, es necesario realizar un estudio más detallado a través de la herramienta de análisis de importancia y gobernabilidad (IGO), para tener una mejor perspectiva sobre cómo abordar estos factores en la definición de los panoramas, estratégicas y proyectos para el desarrollo de la Institución.

Tabla V. Sintesis Matriz de Leopold.

\begin{tabular}{|c|c|c|c|c|c|c|c|c|}
\hline \multirow{2}{*}{\multicolumn{2}{|c|}{ Planeación estratégica }} & \multirow{3}{*}{$\begin{array}{c}\text { Componentes } \\
\text { pestel }\end{array}$} & \multicolumn{6}{|c|}{ Síntesis } \\
\hline & & & \multicolumn{2}{|c|}{ Número de iteraciones } & \multicolumn{4}{|c|}{$\Sigma$} \\
\hline Área & Estrategias del planeación estratégica & & - & + & \multicolumn{2}{|c|}{-} & \multicolumn{2}{|c|}{+} \\
\hline \multirow{6}{*}{ Síntesis } & \multirow{2}{*}{ Número de iteraciones } & - & 22 & & & & & \\
\hline & & + & & 27 & & & & \\
\hline & \multirow{4}{*}{$\Sigma$} & \multirow{2}{*}{ - } & & & 188 & & & \\
\hline & & & & & & 246 & & \\
\hline & & \multirow{2}{*}{+} & & & & & 647 & \\
\hline & & & & & & & & $6 \mathrm{C}$ \\
\hline \multirow{2}{*}{\multicolumn{5}{|c|}{ Promedio del proyecto }} & 8,5 & & 24 & \\
\hline & & & & & & 11 & & 2 \\
\hline
\end{tabular}

Fuente: Los autores, 2018.

Tabla VI. Factores críticos de la prospectiva.

\begin{tabular}{|c|l|l|l|}
\hline Número & \multicolumn{1}{|c|}{ Variable } & \multicolumn{1}{c|}{ Definición } & \multicolumn{1}{c|}{ Estado actual } \\
\hline $\mathbf{1}$ & $\begin{array}{l}\text { Aumento de la } \\
\text { participación en el } \\
\text { mercado }\end{array}$ & $\begin{array}{l}\text { Se refiere a la métrica que hace referencia al } \\
\text { porcentaje de ventas de un negocio en } \\
\text { relación con el total de las ventas de sus } \\
\text { competidores en un mercado específico }\end{array}$ & $\begin{array}{l}\text { En la actualidad no se ha medido } \\
\text { la participación en el mercado de } \\
\text { la Institución }\end{array}$ \\
\hline $\mathbf{2}$ & $\begin{array}{l}\text { Aumento de la } \\
\text { capacidad instalada }\end{array}$ & $\begin{array}{l}\text { Se refiere al crecimiento de las instalaciones, } \\
\text { con el propósito de atender a un mayor } \\
\text { número de pacientes }\end{array}$ & $\begin{array}{l}\text { En mayo se realizó un incremento } \\
\text { de capacidad instalada en } \\
\text { quimioterapia }\end{array}$ \\
\hline
\end{tabular}

Fuente: Los autores, 2018 


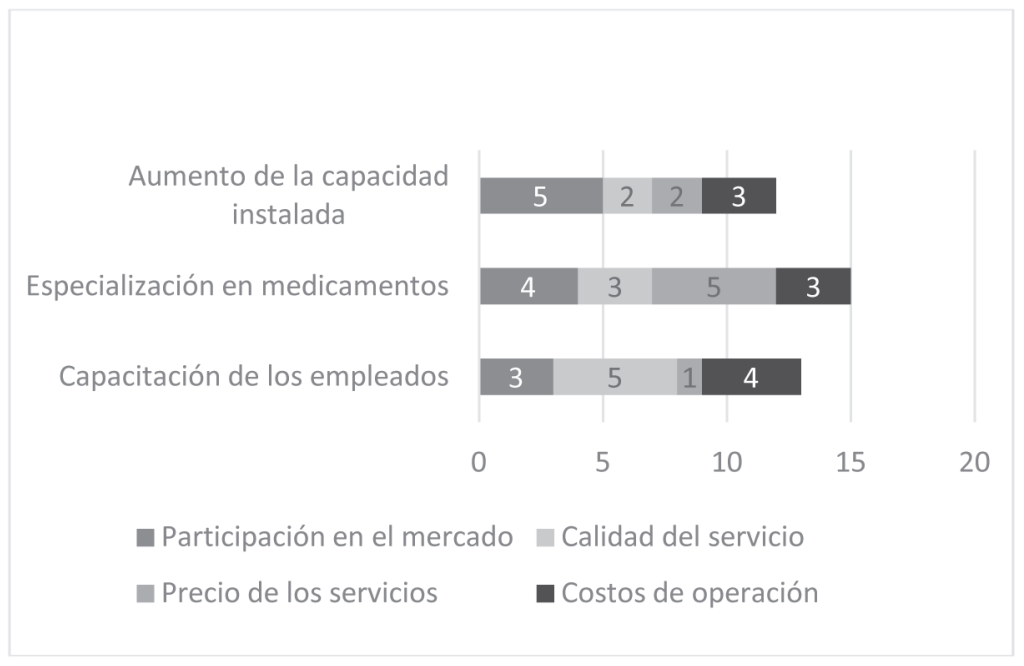

Gráfico 1. Variables tecnológicas.

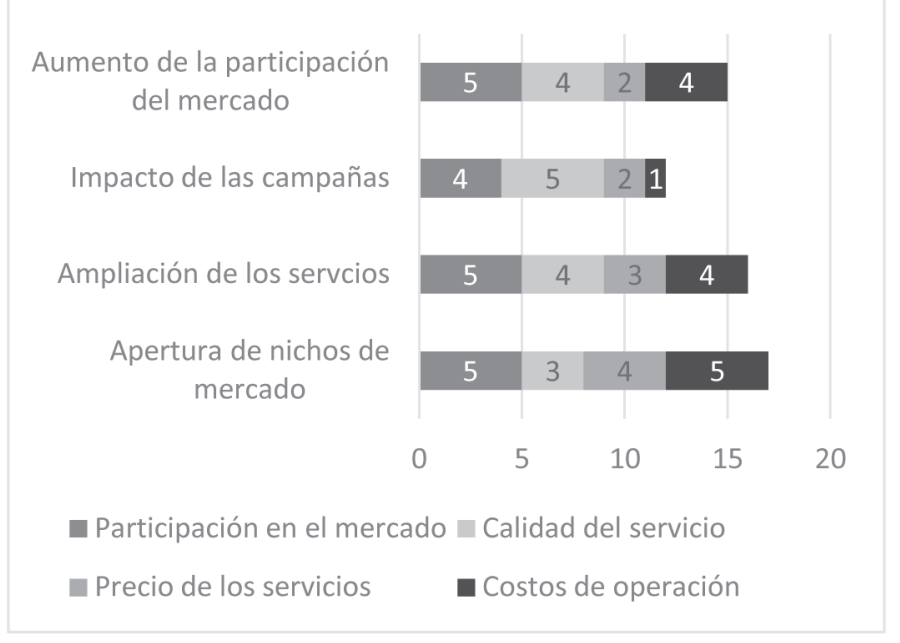

Gráfico 2. Variables no tecnológicas.

La caracterización IGO hace referencia a la importancia y gobernabilidad de cada variable, en este sentido, las variables críticas serán aquellas que tengan la mayor importancia y mayor gobernabilidad dentro del análisis. La importancia tiene que ver con el peso relativo que tiene cada variable para los actores. La gobernabilidad se refiere al nivel de manejo que el sistema tiene sobre la variable. Las dos características se pueden calificar en una escala cualquiera, que permita distinguir cuantitativa y cualitativamente las diferencias entre ellas.

Con los resultados de esta doble caracterización se tendrán cuatro grupos de variables: Alta importancia y alta gobernabilidad, alta importan- cia y baja gobernabilidad, baja importancia y alta gobernabilidad, y baja importancia y baja gobernabilidad. Los resultados obtenidos de la realización del IGO (Importancia y gobernabilidad), se pueden observar en el gráfico 3.

Como se puede evidenciar en el resultado del análisis de importancia y gobernabilidad se refleja la ubicación de factores críticos de acuerdo con la evaluación realizada que sugiere las siguientes prioridades: V1: Aumento de la participación en el mercado, V3: Aumento de la capacidad instalada y V4: Ampliación de los servicios ofrecidos.

Los factores críticos son la base para la identificación de los escenarios futuros para la Institu- 


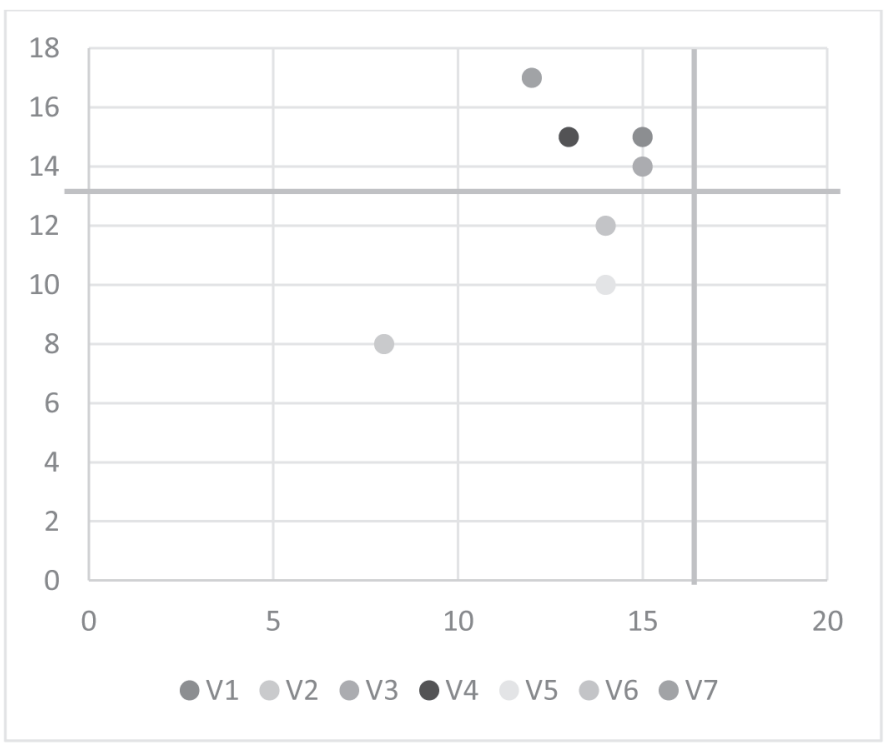

Gráfico 3. Análisis de importancia y gobernabilidad de factores críticos para el sector del tratamiento del cáncer. En 2016 se formularon 1.064 decretos de los cuales el $57 \%$ son considerados no sustanciales.

ción. La definición de los escenarios futuros se centra en la previsibilidad de los factores, es decir, la certeza que se tiene de su comportamiento en el futuro. En este sentido, se deben clasificar, de los 3 factores críticos identificados con la metodología IGO, aquellos que tengan la previsibilidad más baja (incertidumbres), para lo cual se indagó dentro de los diferentes actores y los resultados obtenidos.

La construcción de escenarios se fundamentó en el análisis de los estados futuros mediante una matriz de análisis morfológico, tal como se presenta en la Fig. 1, que consiste en evaluar la posible ocurrencia del estado de una incertidumbre con el estado de otra en un momento dado, dicha reunión de ocurrencia puede variar dependiendo del contexto que se tome como referencia. Para ello se relacionan al lado izquierdo de la matriz los factores más críticos identificados, y al lado derecho se clasifican los estados que consisten en la calificación de la posible ocurrencia de una incertidumbre; para este caso los estados van de uno a tres donde uno es la posición más baja y tres la posición más alta.

El análisis sugiere dos escenarios para el tratamiento y prevención del cáncer de 2019 a 2022 son: Aumento de la capacidad instalada y aumento de los servicios ofrecidos.

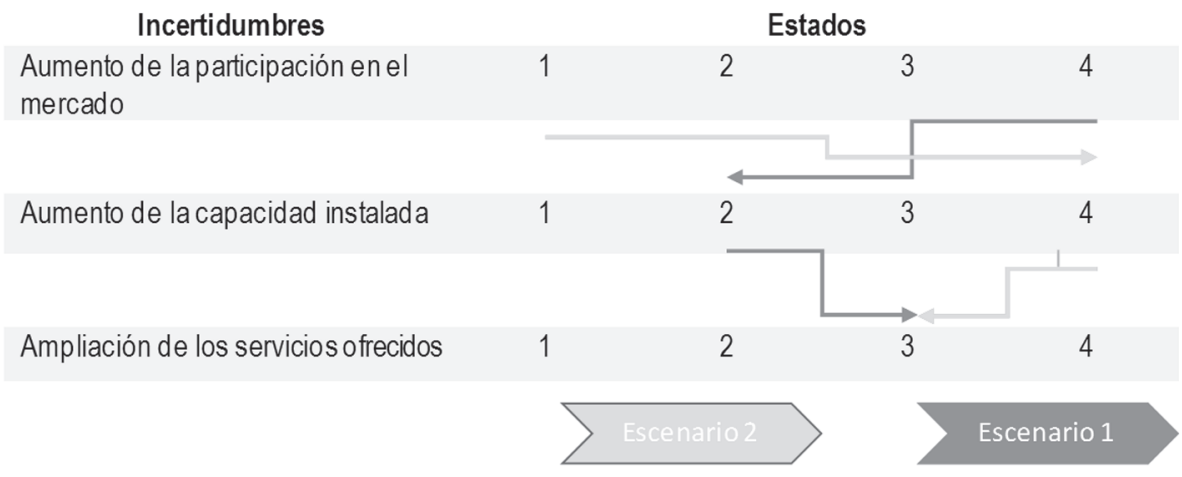

Fig. 1. Matriz de análisis morfológico para la construcción de escenarios futuros. 


\section{Escenario 1:}

\section{Aumento de la capacidad instalada}

Durante el estudio realizado, se concluye que el aumento de la capacidad instalada generaría un mayor beneficio, entre ellos la apertura de nuevos servicios, al tener mayor nivel de atención para los pacientes.

Esto traerá beneficios importantes a la Institución, al poder generar más convenios con las EPS atrayendo gran parte del mercado de pacientes diagnosticados con cáncer. Si se logra aumentar la capacidad instalada, los servicios que se ofertarán serán más completos, incrementando la participación en el mercado.

Como se ha dicho en el estudio, para años posteriores a 2020, el cáncer en mujeres y hombres aumentará en promedio a 80.000 casos para cada género y la Institución podría atender un gran porcentaje de estos y con una alta probabilidad de convertirse en la Institución líder del mercado.

Los pacientes reconocen la calidad y cumplimiento de la institución y el esfuerzo que se está haciendo por ser líderes en el mercado, que acompañado de la ampliación de la capacidad instalada será una oportunidad para liderar en Colombia el diagnóstico y prevención del cáncer.

Los convenios con EPS a nivel nacional harán que la Institución se destaque en el mercado y sea reconocida a nivel nacional, expandiendo sus servicios y generando beneficios que incrementarán sus nichos de mercado.

\section{Escenario 2: \\ Aumento de los servicios ofrecidos}

Con el aumento de los servicios ofrecidos se mejorará la imagen de la Institución, al ofrecer excelentes alternativas para los pacientes diagnosticados con cáncer. Además, podría perfeccionar el nivel de atención y se podría realizar servicios complementarios, que ayuden a la calidad de vida de quien tome el tratamiento.

La Institución se podría destacar por tener un amplio portafolio de servicios, compitiendo con los grandes hospitales en Colombia generando beneficios importantes y destacándose en el sector.
El propósito de aumentar los servicios ofrecidos es incrementar la participación en el mercado. La Institución además debe enfocarse en mantener una atención y un excelente servicio al cliente, generando los menores problemas al paciente teniendo en cuenta su situación de salud y seguir enfocándose en la contratación de especialistas de alta calidad con un personal administrativo eficiente y capacitado.

\section{Plan estratégico}

El plan estratégico que se sugirió a la Institución con ayuda de una empresa externa se basó en la actualización de la misión, visión, objetivos y metas organizacionales, políticas empresariales y valores corporativos hasta el año 2022. Para ello se realizó un taller de comunicación con los empleados, con el fin de aportar ideas y determinar sus inquietudes respecto al funcionamiento actual.

En el aspecto de las metas organizacionales se propuso un plan de trabajo basado en las estrategias generadas que incluyera las actividades, áreas responsables de la Institución, el tiempo y los recursos necesarios para la ejecución de la actividad. El plan se generó para los próximos cuatro años.

\section{Conclusiones}

Se desarrolló un plan estratégico teniendo como pilar importante un estudio de competencias, el cual involucra el marketing y lo hace innovador frente al tema trabajado. Se buscó analizar toda la competencia de la Institución y con ello, lograr sobrepasar las debilidades y volverlas fortalezas y que las amenazas generen oportunidades, con el fin de ser líderes del sector de la prevención y tratamiento del cáncer. Además, con la información recolectada de la competencia, se logró crear estrategias que permitan tener una idea de negocio más consistente, sostenible y con ello traer beneficios tanto para los pacientes como para la Institución y sus empleados.

El mayor aporte del desarrollo del plan estratégico es la prospectiva, la cual sintetiza todas las variables arrojadas por cada uno de los resultados obtenidos en cada una de las herramientas y los clasifica en tecnológicos y no tecnológicos, de es- 
tas variables se evalúa cuál de todas son las más críticas para alcanzar las estrategias generadas del estudio de competencias y de estas generar escenarios, los cuales la Institución puede aplicar en los siguientes 4 años para posicionarse entre las mejores entidades que presten servicios de oncología, teniendo en cuenta la incidencia que tiene el cáncer a nivel nacional y las tendencias del mercado.

\section{REFERENCIAS}

[1] M. Armijo, Manual de planificación estratégica e indicadores de desempeño en el sector público. Disponible en https:/ / www.cepal.org/ilpes/noticias/paginas/3/38453/manual_planificacion_ estrategica.pdf. 2009.

[2] M. Bojórquez, A. Pérez, La planeación estratégica, un pilar en la gestión empresarial. Disponible en https://www.itson.mx/publicaciones/pacioli/ Documents/81/Pacioli-81.pdf. 2013.

[3] Las 10 P's del marketing mix. Reynosablogs. Disponible en https://www.reynosablogs.com/ 2016/07/cuales-son-las-10-p-del-marketing-paramejorar-tu-negocio-servicios-o-productos.html. 2016.

[4] L. Pimentel, Planeación estratégica. Disponible en http:/ / www.unipamplona.edu.co/unipamplona/portallG/home_4/mod_virtuales/modulo5/ 5.2.pdf. 1999.
[5] R. Villafaña, Planeación estratégica. Disponible en http:/ /inn-edu.com/Estrategia/Planestrategica. pdf. 2007.

[6] C. Alvarado, Propuesta para la planeación estratégica de la empresa Aliados Salud Ocupacional LTDA. Universidad de la Salle. 2009.

[7] J. Ballesteros, Diseño de un modelo de Planeación Estratégica para Orlave LTDA. 2015.

[8] Programa Gadex. Análisis PESTEL. Disponible en: http://www.formatoedu.com/web_gades/docs/ 1_Ana_769_lisis_PE.pdf. 2015.

[9] J. Fleitman, Plan de negocios y planeación estratégica empresarial en el siglo XXI. Disponible en https:/ /www.gestiopolis.com/plan-de-negociosy-planeacion-estrategica-empresarial-en-el-sigloxxi/. 2015.

[10] Organización para la cooperación y desarrollo económico, principios de Gobierno Corporativo de la OECD. Disponible en: http:/ / www.confecamaras. org.co/gobierno-corporativo. 2004.

[11] E. Eslava, La prospectiva organizacional. Disponible en: https:// degerencia.com/articulo/que-esprospectiva/. 2010.

[12] F. Bray, Global Cancer Statistics 2018: GLOBOCAN Estimates of Incidence and Mortality Worldwide for 36 Cancers in 185 Countries. 2018.

[13] M. Cerón, Manual de planificación estratégica. Ambato-Ecuador: Universidad Técnica de Ambato. 2007. 
